\title{
Chronic pelvic sepsis with pubic bone osteitis treated with double graciloplasty
}

\author{
N. Michot ${ }^{1} \cdot$ T. Guilbaud $^{1} \cdot$ L. R. Le Nail ${ }^{2} \cdot$ M. Ouaissi ${ }^{1}$ (1)
}

Received: 17 September 2019 / Accepted: 31 October 2019 / Published online: 14 January 2020

(c) Springer Nature Switzerland AG 2020

The patient was a 75-year-old man treated for prostate cancer with radiotherapy in 2006 and radical prostatectomy in June 2016 for recurrence. The tumor was classified as pT3b and Gleason $5+5$. Three months following prostatectomy (September 2016), the patient presented with perineal pain and associated increased prostate-specific antigen. Initial surgical exploration found vesical necrosis under the bladder trigone. A total cystectomy with reimplantation of the ureters on an intestinal loop according to Bricker was, therefore, performed in November 2016.

Postoperatively, the patient presented with chronic sepsis due to pubic bone osteitis. Because of sepsis and tissue loss caused by unsuccessful attempts at pelvic drainage, graciloplasty was performed. This technique has been previously described by Wexner et al. [1] as a treatment for rectourethral, rectovaginal and rectovesical complex fistulas. Our patient had radiotherapy and several open procedures, which is why a double graciloplasty was performed After evacuation of pelvic collections, a first graciloplasty was performed in front of the anal canal, filling the defect facing the pubis, and a second graciloplasty to surround the anal canal, filling the lateral and posterior defects. The procedure is shown in the attached video. The postoperative course was uneventful.

\section{Compliance with ethical standards}

Conflict of interest The authors declare that they have no conflict of interest.

Ethical approval This procedure performed in this study involving human participant was in accordance with the ethical standards of the institutional and/or national research committee and with the 1964 Helsinki declaration and its later amendments or comparable ethical standards.

Informed consent Informed consent was obtained from patient included in the study.

\section{Reference}

1. Ruiz D, Bashankaev B, Speranza J, Wexner SD, Rabau M (2008) Graciloplasty for rectourethral, rectovaginal and rectovesical fistulas: technique overview, pitfalls and complications (2008). Tech Coloproctol 12(3):277-282. https://doi.org/10.1007/s1015 1-008-0433-7

Publisher's Note Springer Nature remains neutral with regard to jurisdictional claims in published maps and institutional affiliations.

Electronic supplementary material The online version of this article (https://doi.org/10.1007/s10151-019-02109-4) contains supplementary material, which is available to authorized users.

M. Ouaissi

m.ouaissi@chu-tours.fr

1 Department of Digestive, Oncological, Endocrine, and Hepatic Surgery, and Hepatic Transplantation, Trousseau Hospital, CHRU Trouseau, Tours, France

2 Department of Traumatology and Orthopedics, Trousseau Hospital, University of Tours, Tours, France 